\title{
Note sur le polymorphisme génétique des lactoprotéines de bovins et de yaks Mongols
}

\author{
F. GROSCLAUDE, Marie-Françoise MAHÉ et J.-P. ACCOLAS * \\ I.N.R.A., Laboratoire de Génétique biochimique, \\ * Laboratoire de Microbiologie laitière et de Génie alimentaire, \\ Centre de Recherches zootechniques, F 78350 Jouy-en-Josas
}

\begin{abstract}
Résumé
Une analyse du polymorphisme génétique des six lactoprotéines principales (quatre caséines, $\alpha$-lactalbumine et $\beta$-lactoglobuline) a été effectuée sur des laits individuels de 145 bovins et de 163 yaks provenant de République Populaire de Mongolie. Le polymorphisme des lactoprotéines du groupe de bovins mongols présente une ressemblance frappante avec celui des races européennes. Il est toutefois montré qu'un variant de caséine $\beta$ de faible fréquence (environ 0,03 ), de même mobilité électrophorétique que le variant $\beta-\mathrm{Cn} \mathrm{A}^{3}$, dérive d'une autre mutation que ce dernier; ce variant a donc reçu une désignation spécifique ( $\beta-\mathrm{Cn} \mathrm{A}^{3}$ Mongolie). Les résultats obtenus dans le groupe de yaks mongols recoupent les conclusions de l'étude effectuée chez des yaks népalais (GROSCLAUDE et al., 1976 a et b), notamment en ce qui concerne l'existence, chez le Yak, d'un variant de $\beta$-lactoglobuline particulier, apparemment fixé ( $\beta$ - $L g$ DYak $)$, et celle des variants $\alpha_{\mathrm{s} 1}$-Cn E et $\alpha_{\mathrm{sz}}-\mathrm{Cn} \mathrm{C}$.
\end{abstract}

\section{Introduction}

L'analyse génétique et biochimique du polymorphisme électrophorétique des principales lactoprotéines du genre Bos, qui s'est avérée riche d'enseignements (Grosclaude, 1979), a été effectuée dans de nombreuses races bovines (Bos taurus), notamment européennes, et dans certaines races ou populations de zébus (Bos indicus) indiennes, africaines et malgaches (voir les revues bibliographiques d'AschafFenBurg, 1968 ; Grosclaude et al., 1973 ; Grosclaude, 1974 et 1979). Pour ce qui est du Yak (Bos grunniens), la seule étude publiée a été effectuée par notre équipe à l'aide d'échantillons de lait collectés au Népal (Grosclaude et al., 1976 a et. b). Malgré le nombre relativement faible d'échantillons disponibles (42 provenant de yaks purs, mais aussi 107 provenant de divers hybrides entre yaks et bovins ou zébus) cette étude avait permis de mettre en évidence et de caractériser un nouveau type de $\beta$-lactoglobuline $\left(\beta-\mathrm{Lg} \mathrm{D}_{\text {Yak }}\right)$, paraissant fixé chez les yaks étudiés, et un variant supplémentaire de caséine $\alpha_{s 1},\left(\alpha_{s 1}-C n E\right)$; elle avait également permis de découvrir, pour la première fois, un polymorphisme de la caséine $\alpha_{\mathrm{s} 2}$, avec en particulier, chez le Yak, un variant d'aspect électrophorétique inhabituel, $\alpha_{\mathrm{s} 2}-\mathrm{Cn} \mathrm{C}$. 
Les recherches dont les résultats sont rapportés dans la présente note ont été entreprises pour tenter de vérifier :

- dans une population bovine implantée très loin de l'aire européenne, le caractère général de nos conclusions sur certains aspects de la génétique des lactoprotéines de Bos taurus,

- dans une population de yaks différente de la population népalaise, la validité de nos principales conclusions sur les lactoprotéines de Bos grunniens.

\section{Matériel et méthodes}

Les échantillons de lait de bovins ont été prélevés, en 1975, dans 5 troupeaux de l'une des circonscriptions (Somon Altan-Bulag) de la province d'Ulan-Bator. Les animaux sont en principe des sujets de la race bovine Mongole, mais on sait que cette race a subi des introductions de reproducteurs importés d'Union Soviétique (Accolas \& Deffontaines, 1975). Les échantillons de lait de yaks ont été prélevés dans 3 troupeaux de la zone montagneuse du Khangaï (province d'Ovör-Khangaï, Somon Dzün - Bayan - Ulaan).

Bien qu'ils aient été additionnés de bichromate de potassium (Grosclaude et al., 1976 a) et congelés après le prélèvement, les échantillons de lait (30 ml environ) sont parvenus coagulés, en raison, sans doute, des délais d'acheminement. Les caséines et les lactosérums ont été préparés par la technique rapide d'Aschaffenburg \& Drewry (1959) ; les lactosérums ont été congelés, et les caséines lyophilisées.

Les électrophorèses, en gel d'amidon et d'acrylamide, ont été effectuées selon les techniques décrites précédemment (Grosclaude et al., 1976 a). Le fait que les laits soient parvenus coagulés ne semble pas avoir affecté les électrophorégrammes de manière sensible sauf, peut-être, au niveau de la caséine $x$ de certains échantillons (voir dans Résultats et Discussion).

La caséine $\beta \mathrm{A}^{3}$ a pu être purifiée par une seule chromatographie sur colonne de CM cellulose 32 selon RichaRdon et al. (1973) à partir d'un mélange (300 mg, préalablement dégraissé dans l'éther) des caséines entières provenant des laits de deux animaux, tous deux de génotype $\beta-\mathrm{Cn}^{\mathrm{A}^{1}} / \beta-\mathrm{Cn}^{\mathrm{A}^{3}}$ au locus $\beta-\mathrm{Cn}$, et homozygotes pour l'allèle $\alpha_{\mathrm{sl}}-\mathrm{Cn}^{\mathrm{B}}$ au locus $\alpha_{\mathrm{s1}}-\mathrm{Cn}$ (colonne de $2 \times 18 \mathrm{~cm}$; gradient linéaire de 0 à $0,32 \mathrm{M}$ de $\mathrm{NaCl}$ avec $2 \times 500 \mathrm{ml}$ de tampon ; débit : $25 \mathrm{ml} / \mathrm{h}$; fractions de $5 \mathrm{ml}$ : la fraction $\beta \mathrm{A}^{3}$ correspondait, dans le chromatogramme, à un épaulement de la partie ascendante du pic de la caséine $\beta$ ). Les techniques d'hydrolyse trypsique et d'électrophorèse sur papier ont été décrites par Grosclaude et al. (1970).

L'estimation, dans le groupe de bovins et dans le groupe de yaks, des fréquences des combinaisons alléliques du groupe de linkage $\alpha_{\mathrm{s} 1}-\mathrm{Cn}, \alpha_{\mathrm{s} 2}-\mathrm{Cn}, \beta-\mathrm{Cn}, \gamma_{-}-\mathrm{Cn}$, a été faite par la méthode de Ceppellinı et al. (1956).

\section{Résultats et Discussion}

Le tableau 1 récapitule l'ensemble des résultats des analyses électrophorétiques. Les fréquences y sont données sans intervalle de confiance, et valent donc seulement pour les deux groupes d'animaux, car il n'est pas certain que ces groupes constituent un échantillon représentatif, stricto sensu, des populations dont ils sont issus. 


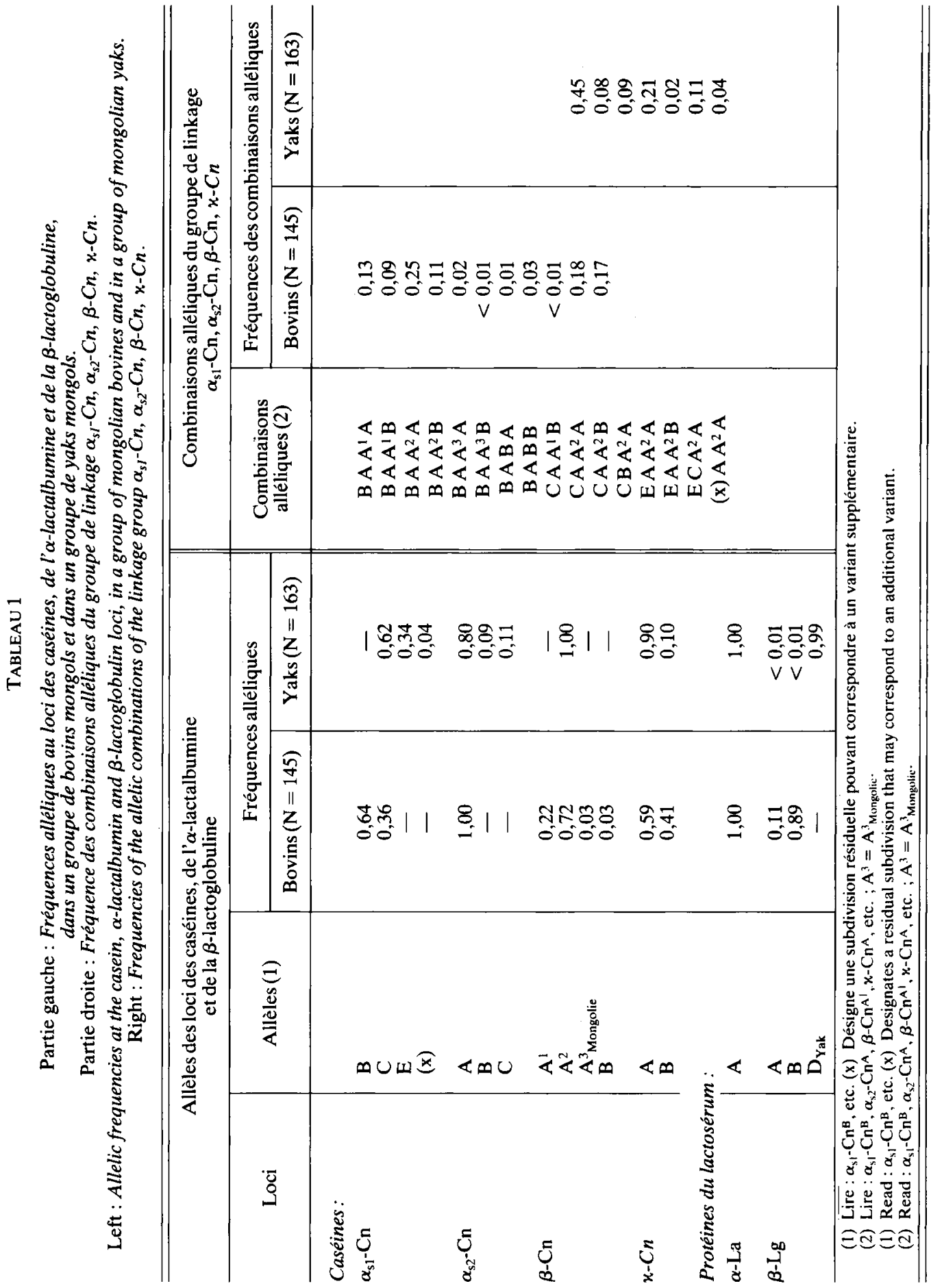




\section{A. Bovins mongols}

L'électrophorèse des laits de bovins mongols révèle des caractéristiques qui ne se distinguent pas de celles des races européennes. C'est ainsi que les loci $\alpha_{\mathrm{s} 2}$-Cn et $\alpha$-La sont monomorphes, et que l'on retrouve, aux autres loci, les variants bovins principaux, avec des fréquences qui sont dans la norme de celles observées dans les races européennes. En particulier, comme dans la quasi-totalité des races bovines étudiées jusqu'ici, la fréquence de l'allèle $\alpha_{s 1}-\mathrm{Cn}^{\mathrm{B}}$ est supérieure à celle de l'allèle $\alpha_{\mathrm{s} 1}-\mathrm{Cn}^{\mathrm{C}}$, à l'inverse de ce qui s'observe chez les zébus. Par ailleurs, les allèles $\beta-\mathrm{Cn}^{\mathrm{A}^{1}}$ et $\beta-\mathrm{Cn}^{\mathrm{B}}$ sont, ici aussi, transmis avec l'allèle $\alpha_{\mathrm{s} 1}-\mathrm{Cn}^{\mathrm{B}}$ et non avec l'allèle $\alpha_{\mathrm{s} 1}-\mathrm{Cn}^{\mathrm{C}}$, à une rare exception près.

Toutefois, la présence à faible fréquence, chez les bovins mongols, de l'allèle $\beta-\mathrm{Cn}^{\mathrm{A}^{3}}$, qui n'a été observé dans les races françaises, à faible fréquence également, que dans deux cas (Normande et Holstein-Pie-Noire), et surtout le fait que cet allèle soit, dans la race Mongole, associé à $\alpha_{\mathrm{sI}}-\mathrm{Cn}^{\mathrm{B}}$, et non à $\alpha_{\mathrm{sI}}-\mathrm{Cn}^{\mathrm{C}}$ comme dans les deux races françaises, représentait un élément inattendu. Cet élément nous a conduits à entreprendre une analyse biochimique partielle du variant $\beta-C_{n} \mathrm{~A}^{3}$ mongol.

La substitution d'acides aminés spécifique du variant $\beta-\mathrm{Cn} \mathrm{A}^{3}$ européen est une substitution $106 \mathrm{His} \rightarrow \mathrm{Gln}$. Les effets de cette substitution peuvent être facilement mis en évidence par électrophorèse sur papier d'un hydrolysat trypsique de caséine $\beta$. En effet, dans le variant de référence $\beta-\mathrm{Cn} \mathrm{A}^{2}$, le résidu His 106 appartient à un dipeptide trypsique His-Lys très mobile à $\mathrm{pH} \mathrm{4,7} \mathrm{et} \mathrm{donnant} \mathrm{avec} \mathrm{le} \mathrm{réactif} \mathrm{de} \mathrm{Pauly}$ la coloration spécifique de l'histidine. Dans le variant $\beta-\mathrm{Cn} \overline{\mathrm{A}}^{3}$, la substitution $106 \mathrm{His} \rightarrow \mathrm{Gln}$ réduit la mobilité du dipeptide considéré, qui, en outre, ne se colore plus avec le réactif de Pauly (Ribadeau-Dumas et al., 1970).

Une petite quantité de fraction $\beta A^{3}(35 \mathrm{mg})$ a pu être purifiée par chromatographie sur $\mathrm{CM}$ cellulose 32 comme indiqué dans "Matériel et Méthodes». Un hydrolysat trypsique de cette fraction $\left(5 \mathrm{mg} ; \mathrm{E} / \mathrm{S}=1 / 50 ; 3 \mathrm{~h} 30 ; 37^{\circ} \mathrm{C}\right)$ a été soumis à une

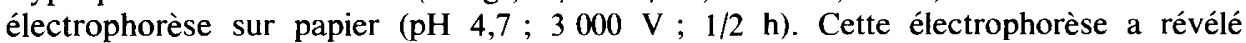
la présence du peptide caractéristique His-Lys. Ce résultat indique que la substitution spécifique du variant de type $\mathrm{A}^{3}$ observé chez les bovins mongols n'est pas la même que celle du variant européen. Ce variant, découvert en Mongolie, a donc été désigné par $\beta$-Cn $\mathrm{A}^{3}{ }_{\text {Mongolie }}$ L'allèle qui le contrôle dérive aussi d'une autre mutation que celle ayant donné l'allèle $\beta-\mathrm{Cn}^{\mathrm{A}^{3}}$ européen. Par manque de caséine, l'analyse du variant $\beta-\mathrm{Cn} \mathrm{A}^{3}$ Mongolie n'a pu être poussée plus avant.

\section{B. Yaks mongols}

On retrouve, au locus $\beta$-Lg, le variant $\beta$-Lg $D_{\text {Yak }}$, découvert au Népal, dont l'allèle peut être considéré comme fixé, en dépit de la présence, à très faible fréquence, des allèles $\beta-\mathrm{Lg}^{\mathrm{A}}$ et $\beta-\mathrm{Lg}^{\mathrm{B}}$; cette présence peut en effet être interprétée, avec beaucoup de vraisemblance, comme une trace de l'hybridation qui est pratiquée, entre yaks et bovins, par les éleveurs locaux (Accolas \& Deffontaines, 1975).

On retrouve également, au locus $\alpha_{\mathrm{s1}}-\mathrm{Cn}$, à côté du variant $\alpha_{\mathrm{s1}}-\mathrm{Cn} \mathrm{C}$, dont l'allèle prédomine, le variant $\alpha_{\mathrm{s} 1}-\mathrm{Cn} \mathrm{E}$ découvert au Népal (Grosclaude et al., 1976 a). Nous avions par ailleurs attiré l'attention, dans le même travail, sur l'existence possible d'un autre variant de caséine $\alpha_{\mathrm{sl}}$, de mobilité légèrement plus anodique, à $\mathrm{pH}$ alcalin, 
que le variant $\alpha_{s 1}-C_{n} C$. La même observation a été faite chez les yaks mongols. Toutefois, en raison de l'insuffisance des données génétiques et biochimiques, il ne peut être conclu en toute rigueur à l'existence de ce variant, même si elle paraît vraisemblable. La fraction correspondante a été notée $(x)$ dans le tableau 1. De manière générale, les fréquences alléliques au locus $\alpha_{\mathrm{st}}-\mathrm{Cn}$ dans le groupe mongol sont remarquablement voisines de celles observées au Népal.

Dans une dizaine de laits individuels, une bande de caséine $x$ notée comme $x-\mathrm{Cn} \mathrm{A}$ présentait en fait une mobilité un peu plus élevée que celle du variant $x$-Cn A. Il n'a pas été possible d'établir si ce phénomène était dû à l'existence d'un variant supplémentaire, en l'occurrence peu fréquent, ou à une altération de la caséine $x$, liée au délai d'acheminement des échantillons.

C'est au locus $\alpha_{\mathrm{s}_{2}}-\mathrm{Cn}$ qu'apparaît la seule différence notable avec les résultats obtenus au Népal. En effet, si le variant $\alpha_{s 1}-C n C$ découvert au Népal se retrouve bien dans le groupe de yaks mongols, on observe également, dans ce dernier, le variant $\alpha_{\mathrm{s} 1}-\mathrm{Cn} \mathrm{B}$, qui, au Népal, paraissait propre aux bovins et aux zébus (Grosclaude et al., 1976 b). Cette observation peut amener à considérer différemment les relations entre les variants $\alpha_{\mathrm{s} 2}$ - $\mathrm{Cn} \mathrm{A}$, B et C (MahÉ \& Grosclaude, 1982).

En ce qui concerne les combinaisons alléliques du groupe de linkage formé par les loci des caséines, on constate que l'allèle $\alpha_{\mathrm{s} 2}-\mathrm{Cn}^{\mathrm{C}}$ est associé, dans le groupe de yaks mongols, à l'allèle $\alpha_{s 1}-\mathrm{Cn}^{\mathrm{E}}$, alors que sur la base d'observations faites chez des femelles népalaises hybrides (yak $\times$ zébu) $\alpha_{\mathrm{s} 2}-\mathrm{Cn}^{\mathrm{C}}$ paraissait associé à $\alpha_{\mathrm{s} 1}-\mathrm{Cn}^{\mathrm{C}}$. En fait chez l'un au moins des yaks purs népalais, $\alpha_{\mathrm{s} 2}-\mathrm{Cn}^{\mathrm{C}}$ était également associé à $\alpha_{\mathrm{s} 1}-\mathrm{Cn}^{\mathrm{E}}$. L'ensemble de ces résultats permet de conclure que $\alpha_{\mathrm{s} 2}-\mathrm{Cn}^{\mathrm{C}}$ est associé aussi bien à $\alpha_{\mathrm{sl}}-\mathrm{Cn}^{\mathrm{C}}$ qu'à $\alpha_{\mathrm{sI}}-\mathrm{Cn}^{\mathrm{E}}$.

La prédominance, au locus $\alpha_{s_{1}}-\mathrm{Cn}$ du Yak, de l'allèle $\alpha_{s 1}-C_{n}{ }^{C}$ est confirmée par les données du tableau 1. Ceci contribue à étayer l'hypothèse selon laquelle cet allèle représenterait la forme originelle au locus $\alpha_{\mathrm{s} 1}-\mathrm{Cn}$ dans le genre Bos (Grosclaude, 1979). Dans ce domaine des relations phylogéniques au sein du genre Bos, rappelons que Bell et al. (1981 b) ont trouvé chez le Banteng (Bos javanicus) un type de $\beta$-lactoglobuline, appelé $\beta$ - $\mathrm{Lg} \mathrm{E}$, qui sur la base d'une analyse biochimique, paraît identique au variant $\beta$-Lg $D_{Y a k}$. Cette observation pose évidemment en termes nouveaux le problème des relations entre le Yak et les autres espèces du genre Bos. Il nous paraît par contre prématuré, au vu des données existantes, de conclure avec Bell et al. (1981 a), à l'identité du variant du Yak $\alpha_{\mathrm{sl}}-\mathrm{Cn} \mathrm{E}$ avec le type $\alpha_{\mathrm{sl}}-\mathrm{Cn} \mathrm{E}_{\mathrm{Bali}}$ également observé par ces auteurs chez le Banteng.

Reçu pour publication en novembre 1982.

\section{Remerciements}

Nous sommes très reconnaissants aux Autorités Mongoles, en particulier à l'Ambassade de la République Populaire de Mongolie à Paris de leur compréhension. Nous remercions également tous ceux qui ont contribué à nous faire parvenir les échantillons de lait. 


\section{Summary}

\section{A note on the genetic polymorphism of the lactoproteins of Mongolian Bovines and Yaks}

A study of the genetic polymorphism of the six main lactoproteins (four caseins, $\alpha$-lactalbumin and $\beta$-lactoglobulin) has been carried out on 145 bovine and 163 yak milks from the People's Republic of Mongolia. The polymorphism of the lactoproteins of the Mongolian bovines shows a striking resemblance to that observed in European breeds. Nevertheless a low frequency $(0.03)$ $\beta$ casein variant with the same electrophoretic mobility as $\beta-C n A^{3}$ was shown to derive from a mutation other than that determining $\beta-\mathrm{Cn} \mathrm{A}^{3}$; this variant was thus given a specific designation ( $\beta-\mathrm{Cn} \mathrm{A}^{3}$ Mongolie). The observations made on Mongolian yaks concord well with the conclusions of the study previously carried out on Nepalese yaks (GROSCLAUDE et al., 1976 a and b) concerning the existence, in the Yak, of the single $\beta$-lactoglobulin type $\beta-\mathrm{Lg} \mathrm{D}_{Y a k}$ and the occurrence of the variants $\alpha_{\mathrm{s} 1}-\mathrm{Cn} \mathrm{E}$ and $\alpha_{\mathrm{s2}}-\mathrm{Cn} \mathrm{C}$.

\section{Références bibliographiques}

Accolas J.-P., Deffontaines J.-P., 1975. Les activités rurales en République Populaire de Mongolie. I. - Agriculture et Elevage. Etudes Mongoles, Cahier du Centre d'Etudes mongoles, Laboratoire d'Ethnologie, Université de Paris X - Nanterre, 6, 9-53.

AsChafFendurg R., 1968. Genetic variants of milk proteins; their breed distribution. J. Dairy Res., 35, 447-460.

Aschaffenburg R., Drewry J., 1959. New procedure for the routine determination of the various non casein proteins in milk. $X V^{\mathrm{e}}$ Cong. Inter. Lait, 3, sect. 5, 1631-1637.

Bell K., HoPPER K.E., Mc KenZIE H.A., 1981 a. Bovine $\alpha$-lactalbumin $C$ and $\alpha_{\mathrm{st}^{-}}, \beta$ - and $x$-caseins of Bali (Banteng) Cattle, Bos (Bibos) javanicus. Aust. J. Biol. Sci., 34, 149-159.

Bell K., Mc Kenzie H.A., Shaw D.C., 1981 b. Bovine $\beta$-Lactoglobulin E, F and G of Bali (Banteng) Cattle, Bos (Bibos) javanicus. Aust. J. Biol. Sci., 34, 133-147.

Ceppellini R., Siniscalco M., Smith C.A.B., 1956. The estimation of gene frequencies in a random-mating population. Ann. hum. Genet., 20, 97-115.

Grosclaude F., 1974. Analyse génétique et biochimique du polymorphisme électrophorétique des caséines $\alpha_{s l}, \beta$ et $x$ chez les bovins (Bos taurus) et les zébus (Bos indicus). Thèse de Doctorat d'Etat ès-Sciences, Paris.

GrosClaude F., 1979. Polymorphism of milk proteins : some biochemical and genetical aspects. Proc. 16th Int. Conf. Anim. Blood Grps (Leningrad, 1978), 1, 54-92.

Grosclaude F., Mahé Marie-Françoise, Mercier J.-C., Bonnemaire J., Teissier J.-H., 1976 a. Polymorphisme des lactoprotéines de bovinés népalais. I. - Mise en évidence, chez le yak, et caractérisation biochimique de deux nouveaux variants : $\beta$-lactoglobuline $D_{\text {yak }}$ et caséine $\alpha_{\mathrm{s} 1} \mathrm{E}$. Ann. Génét. Sél. anim., 8, 461-479.

Grosclaude F., Mahé Marie-Françoise, Mercier J.-C., Bonnemaire J., Teissier J.-H., 1976 b. Polymorphisme des lactoprotéines de bovinés népalais. II. - Polymorphisme des caséines « $\alpha_{\mathrm{s}}$-mineures »; le locus $\alpha_{\mathrm{s} 2}-\mathrm{Cn}$ est-il lié aux loci $\alpha_{\mathrm{s} 1}-\mathrm{Cn}, \beta-\mathrm{Cn}$ et $x_{-} \mathrm{Cn}$ ? Ann. Génét. Sél. anim., 8, 481-491.

Grosclaude F., Mercier J.-C., Ribadeau-Dumas B., 1970. Structure primaire de la caséine $\alpha_{s 1}$ bovine. Localisation des peptides trypsiques dans les fragments obtenus par hydrolyse trypsique de la caséine maléylée. Eur. J. Biochem., 14, 98-107.

Grosclaude F., Mercier J.-C., Ribadeau-Dumas B., 1973. Genetic aspects of cattle casein research. Neth. Milk Dairy J., 27, 328-340.

Mahé Marie-Françoise, Grosclaude F., 1982. Polymorphisme de la caséine $\alpha_{\mathrm{s} 2}$ des bovinés : caractérisation du variant $C$ du yak (Bos grunniens). Ann. Génét. Sél. anim., 14, 339-354.

Ribadeau-Dumas B., Grosclaude F., Mercier J.-C. Localisation, dans la chaîne peptidique de la caséine $\beta$ bovine, de la substitution His/Gln différenciant les variants génétiques $\mathrm{A}^{2}$ et $\mathrm{A}^{3}$. C.R. Acad. Sc. Paris, 270, 2369-2372.

Richardson B.C., Creamer L.K., Munford R.E., 1973. Comparative micelle structure. I. - The isolation and chemical characterisation of caprine $x$-casein. Biochim. Biophys. Acta, 310, 111-117. 